\title{
Studies on Dielectric and Conductive Properties of Polymeric Charge-Transfer Complexes. II. TCNQ Salts of Block Polymers Containing Polycation Sequences ${ }^{\dagger}$
}

\author{
Shinobu IKeno,* Masaaki Yokoyama, and Hiroshi Mikawa \\ Department of Applied Chemistry, Faculty of Engineering, \\ Osaka University, Suita, Osaka 565, Japan.
}

(Received March 31, 1977)

\begin{abstract}
The synthesis of several new TCNQ (tetracyanoquinodimethane) salts of block ionene polymers where polycation sequences are broken by copolymerizing a long sequence of a polyether prepolymer, and their conductive and dielectric properties are reported. The polymers obtained are flexible and have improved mechanical properties. The complex TCNQ salts exhibit low specific resistivity (about $10^{4} \Omega \mathrm{cm}$ ) and a high dielectric constant (about $10^{3}$ ) at room temperature. The dispersion of the dielectric constant is observed in all polymers and the activation energies for the polarization process agree very closely with those of electrical conduction. These electrical properties of TCNQ salts of block ionene polymers can be properly explained in terms of the new structural model previously proposed for the TCNQ salts of ionene polymers. In the block polycation-TCNQ salts, TCNQ molecules are packed randomly in the complex polycation network and form conduction paths which differ from hitherto considered long columns with face-to-face stacked TCNQ molecules along the polymer chain, because the polycation sequences are broken by the introduction of polyether prepolymer units. Taking the conduction paths of short isolated ones as the microscale conducting particles dispersed in an insulating matrix, the dielectric property of the polymers is qualitatively explained by the Maxwell-Wagner type polarization.

KEY WORDS Polycation-TCNQ Salt/Block Copolymer/Simple Salt / Complex Salt / Dielectric Constant / Tan $\delta$ / Conductivity / Polarization / Maxwell-Wagner Polarization / Doping /
\end{abstract}

A great need for electrically conducting polymers which are flexible and capable of being shaped by ordinary processes, has stimulated recent researches. ${ }^{1,2}$ Also, intensive studies on the conductive properties of polycation-TCNQ (tetracyanoquinodimethane) salts ${ }^{3,4}$ have been carried out. However, little attention has been paid to the dielectric properties of these materials.

In a previous work, ${ }^{5}$ we studied the dielectric properties of TCNQ salt of a polycation having cationic sites in the main chain, in connection with their conductive properties, and found that (1) these polymeric-TCNQ salts exhibited very high dielectric constant, and (2) the polarization could

\footnotetext{
+ Studies on the Polymers Having High Dielectric Constant. III. Part II, ref 5.

* On leave of absence from Research Division, Matsushita Electric Works Co, Ltd., Kadoma, Osaka.
}

be essentially explained by the Maxwell-Wagner type polarization, induced by mobile electronic carriers in the isolated conductive phase. We proposed a new structural model which could explain the main features of these electrical properties in polymers of this kind.

In this work, as an attempt to attain a material such that all of the conductive polycationTCNQ portion is completely isolated by the insulating portion, block copolymers composed of a conductive portion of oligocation-TCNQ salt and an insulating portion of a polyether prepolymer have been synthesized, and their electrical properties have been investigated.

\section{EXPERIMENTAL}

\section{Synthesis of the Polymers}

The synthetic scheme of the polymers is il- 
Step-1 Synthesis of demethylamino-end capped prepolymer (II)

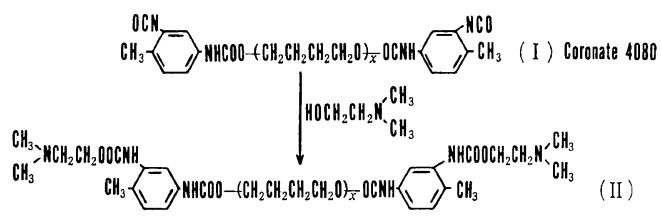

Step-2 Synthesis of $\alpha$, $\omega$-dichloro ionene oligomer (III)

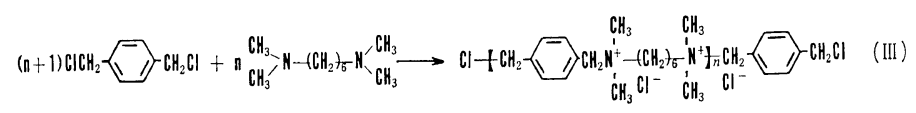

Step-3 Synthesis of block ionene polymer (IV)

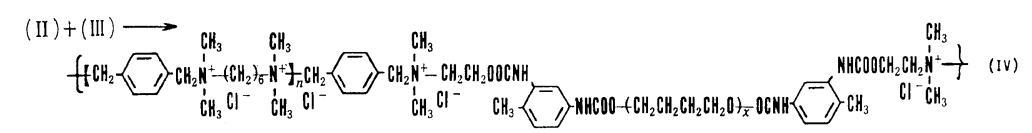

Step-4 Synthesis of TCNQ salt of block ionene polymer (Simple salt (V))

(IV) + LiTCNQ $\longrightarrow$ Simple salt (V)

Step-5 Doping of neutral TCNQ molecules into simple salt (Formation of complex salt (VI))

$$
\text { (V) }+\mathrm{TCNQ}^{\circ} \longrightarrow \text { Complex salt (VI) }
$$

Figure 1. Synthetic scheme of block ionene polymers, and their TCNQ salts.

lustrated in Figure 1.

Synthesis of the Block Ionene Polymers (IV).

Coronate-4080 from Japan Polyurethan Industry was used as isocyanate-end capped prepolymer (I), and dimethylamino-end capped prepolymer (II) was synthesized according to the method in the literature. ${ }^{6}$ The molecular weight of (II) was found to be 3350 , based on the amine titration. $^{7} \quad \alpha, \omega$-Dichloro ionene oligomers (III) of average block sequences of $n=5$ and 10 were prepared in a similar manner described in the literature. ${ }^{4}$ In the following, the block ionene polymers (IV) prepared by the reaction of (II) and (III) are abbreviated as BI- $n 5$ and BI- $n 10$, corresponding to the average block sequences of (III), respectively.

Taking BI- $n 5$ as an example, the synthesis of block ionene polymers is as follows: In $10 \mathrm{~m} l$ of dimethylformamide (DMF), $p$-bis(chloromethyl) benzene $(2.0964 \mathrm{~g})$ was dissolved, and to this, a solution of $N, N, N^{\prime}, N^{\prime}$-tetramethylhexamethylene diamine $(1.7193 \mathrm{~g})$ in $10 \mathrm{ml}$ of methanol was added with stirring at room temperature, and the solution was maintained at $50^{\circ} \mathrm{C}$ for $2 \mathrm{hr}$. At the end of the reaction, $18 \mathrm{~m} l$ of methanol was added to the reaction mixture to make the solution clear. To this solution was added dimethylamino-end capped prepolymer $(6.6987 \mathrm{~g})$ which had been dissolved in a mixed solvent of $3 \mathrm{~m} l$ of DMF and $12 \mathrm{~m} l$ of methanol, and the dropping funnel was washed with a mixed solvent of $3 \mathrm{ml}$ of DMF and $27 \mathrm{~m} l$ of methanol. The solution was maintained at $60^{\circ} \mathrm{C}$ for $20 \mathrm{hr}$. The polymer was precipitated and purified by pouring the polymer solution into a mixed solvent of benzene and $n$-hexane; yield, $8.80 \mathrm{~g}(83.8 \%)$. Anal. Calcd for BI- $n 5(n=5$, $x=39): \mathrm{C}, 63.83 ; \mathrm{H}, 9.87 ; \mathrm{N}, 4.25 ; \mathrm{Cl}, 8.07$. Found: C, 61.81; H, 9.65; N, 4.96; Cl, 8.49.

Synthesis of TCNQ Salts of Block Ionene Polymers (Simple Salt (V)). The simple salts (V) were prepared by the reaction of $1.3 \mathrm{~mol}$ of LiTCNQ per mol of $\mathrm{N}^{+}$of the block ionene polymers. A solution of BI- $n 5(1.9904 \mathrm{~g})$ in $54 \mathrm{~m} l$ of methanol was added dropwise into a solution of LiTCNQ $(1.2431 \mathrm{~g})$ in $87 \mathrm{~m} l$ of methanol with stirring at room temperature in nitrogen atmosphere. A simple salt was precipitated immediately. After stirring for $2 \mathrm{hr}$ in a stream of nitrogen, this precipitate was centrifuged and washed with methanol until the washings were 
colorless, and dried in a vacuum; yield $2.43 \mathrm{~g}$ (84.6\%).

Anal. Calcd for $100-\%$ substitution: C, 69.82 ; H, 7.79; N, 12.29. Found: C, 68.90; H, 7.52; N, 12.94; Cl, 0.69 .

Doping of Neutral TCNQ molecules into Simple Salt (Formation of Complex Salt (VI)). Weighed amount of $\mathrm{TCNQ}^{\circ}$ and simple salt $(\mathbf{V})$ were mixed in purified DMF, and films of the complex salts were obtained by casting this solution onto a glass plate and evaporating the solvent under vacuum.

\section{Samples and Electrical Measurements}

Polymer solution was casted on a glass plate and the solvent was evaporated under vacuum. After taking off the polymer films from the glass plate, the films were throughly dried under vacuum at room temperature. The thickness of the films was from $100 \mu \mathrm{m}$ to $250 \mu \mathrm{m}$. Gold was evaporated onto both sides of the films to make two terminal electrodes. Measurements of electrical properties were made accoding to methods described in a previous paper. ${ }^{5}$ Temperature dependence of the resistivity was measured with a rise in temperature of approximately $2^{\circ} \mathrm{C} / \mathrm{min}$ at a constant voltage.

\section{RESULTS AND DISCUSSION}

\section{General Properties of the Polymers}

The obtained block ionene polymers (IV) are easily soluble in methanol and DMF. The films of the block copolymer (IV) are flexible and tough, while those of the ionene polymer ${ }^{5}$ from $N, N, N^{\prime}$, $N^{\prime}$-tetramethyl-1, 6-hexamethylenediamine and $p$ bis(chloromethyl)benzene is brittle in the dry state. Thus, an improvement in the mechanical properties of these polymers is attained through introduction of a flexible polyether unit into the main chain of the ionene type polymers. The rigidity of the polymers (IV) increases with increasing sequence length of the ionene oligomers (III). The simple TCNQ salts of BI- $n 5$ and BI- $n 10$ are also easily soluble in amide solvents such as DMF and DMAc, and form tough, flexible and highly colored films. In the following, the symbol (S) represents simple salts. Doping with neutral TCNQ $\left(\mathrm{TCNQ}^{\circ}\right)$ into the simple TCNQ salts of block ionene polymers increases the rigidity of the films, and makes the surface of films mat, while the surface of the films of simple salts is lustrous.

The anion exchange reaction between block

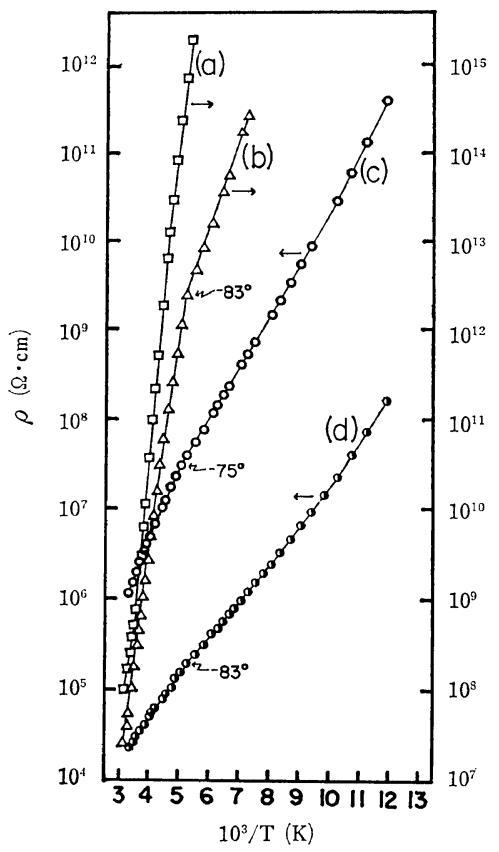

Figure 2. Plots of $\log$ resistivity $v s .10^{3} / T$ for $\mathrm{BI}-n 5$ in chloride form, its simple salt and complex salt: (a), in chloride form; (b), simple salt; (c), complex salt (doping ratio, 0.20); (d), complex salt (doping ratio, $0.50)$.

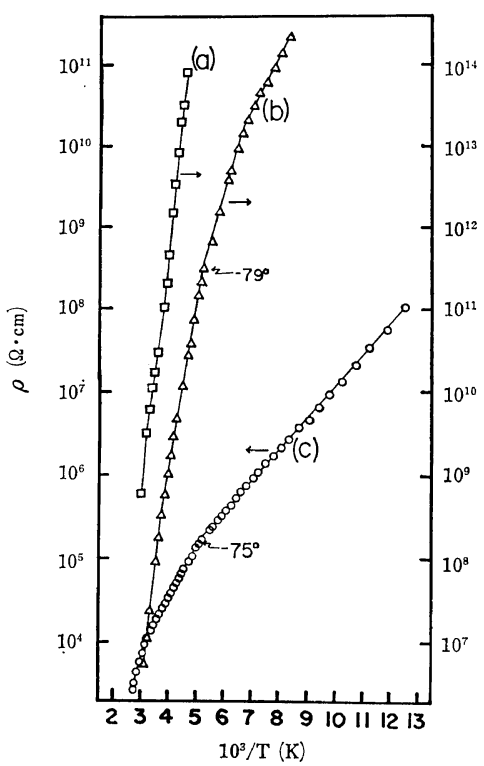

Figure 3. Plots of $\log$ resistivity $v s .10^{3} / T$ for $\mathrm{BI}-n 10$ in chloride form, its simple salt and complex salt: (a), in chloride form; (b), simple salt; (c), complex salt (doping ratio, 0.50). 


\section{S. IKeno, M. Yokoyama, and H. MiKawa}

Table I. Specific resistivities $(\rho)$ and activation energies $(\Delta E)$ of block ionene polymers and their simple and complex salts

\begin{tabular}{|c|c|c|c|c|c|}
\hline Sample & $\begin{array}{l}\text { TCNQ, } \\
\text { wt } \%^{a}\end{array}$ & $\rho_{25^{\circ} \mathrm{C}}$ & $\rho_{-50^{\circ} \mathrm{C}}$ & & eV \\
\hline $\mathrm{BI}-n 5$ & 0 & $5.6 \times 10^{8}$ & $3.6 \times 10^{12}$ & $\begin{array}{l}0.56\left(-88^{\circ}--57^{\circ} \mathrm{C}\right) \\
0.42\left(6^{\circ}-43^{\circ} \mathrm{C}\right)\end{array}$ & $0.79\left(-57^{\circ}-6^{\circ} \mathrm{C}\right)$ \\
\hline $\mathrm{BI}-n 5(\mathrm{~S})$ & 35 & $1.8 \times 10^{8}$ & $6.0 \times 10^{10}$ & $\begin{array}{ll}0.20 & \left(-140^{\circ}--83^{\circ} \mathrm{C}\right) \\
0.51 & \left(-39^{\circ}-40^{\circ} \mathrm{C}\right)\end{array}$ & $0.44\left(-83^{\circ}--39^{\circ} \mathrm{C}\right)$ \\
\hline $\mathrm{BI}-n 5(0.20)^{\mathrm{b}}$ & 40 & $1.7 \times 10^{6}$ & $1.3 \times 10^{7}$ & $\begin{array}{ll}0.13 & \left(-189^{\circ}--178^{\circ} \mathrm{C}\right) \\
0.11 & \left(-149^{\circ}--75^{\circ} \mathrm{C}\right) \\
0.18 & \left(-36^{\circ}-25^{\circ} \mathrm{C}\right)\end{array}$ & $\begin{array}{ll}0.12 & \left(-178^{\circ}--149^{\circ} \mathrm{C}\right) \\
0.15 & \left(-75^{\circ}--36^{\circ} \mathrm{C}\right)\end{array}$ \\
\hline $\mathrm{BI}-n 5(0.50)$ & 45 & $2.9 \times 10^{4}$ & $9.1 \times 10^{4}$ & $\begin{array}{l}0.10\left(-189^{\circ}--178^{\circ} \mathrm{C}\right) \\
0.078\left(-149^{\circ}--83^{\circ} \mathrm{C}\right)\end{array}$ & $\begin{array}{l}0.087\left(-178^{\circ}--149^{\circ} \mathrm{C}\right) \\
0.096\left(-83^{\circ}-25^{\circ} \mathrm{C}\right)\end{array}$ \\
\hline $\mathrm{BI}-n 10$ & 0 & $2.0 \times 10^{10}$ & $3.1 \times 10^{13}$ & $\begin{array}{ll}0.50 & \left(-79^{\circ}--50^{\circ} \mathrm{C}\right) \\
0.47 & \left(-9^{\circ}-35^{\circ} \mathrm{C}\right)\end{array}$ & $\begin{array}{l}0.74 \quad\left(-50^{\circ}-9^{\circ} \mathrm{C}\right) \\
0.90\left(35^{\circ}-54^{\circ} \mathrm{C}\right)\end{array}$ \\
\hline $\mathrm{BI}-n 10(\mathrm{~S})$ & 43 & $5.3 \times 10^{7}$ & $1.1 \times 10^{10}$ & $\begin{array}{ll}0.14 & \left(-154^{\circ}--124^{\circ} \mathrm{C}\right) \\
0.42 & \left(-79^{\circ}--3^{\circ} \mathrm{C}\right)\end{array}$ & $\begin{array}{ll}0.25 & \left(-124^{\circ}-79^{\circ} \mathrm{C}\right) \\
0.61 & \left(-3^{\circ}-50^{\circ} \mathrm{C}\right)\end{array}$ \\
\hline $\mathrm{BI}-n 10(0.50)$ & 54 & $1.6 \times 10^{4}$ & $6.3 \times 10^{4}$ & $\begin{array}{l}0.077\left(-193^{\circ}-75^{\circ} \mathrm{C}\right) \\
0.22\left(35^{\circ}-91^{\circ} \mathrm{C}\right)\end{array}$ & $0.12\left(-75^{\circ}-35^{\circ} \mathrm{C}\right)$ \\
\hline Ionene $(\mathbf{S})^{\mathrm{c}}$ & 60 & $2.5 \times 10^{7}$ & $9.0 \times 10^{9}$ & $0.22\left(-135^{\circ}--83^{\circ} \mathrm{C}\right)$ & $0.41 \quad\left(-83^{\circ}-60^{\circ} \mathrm{C}\right)$ \\
\hline Ionene $(0.40)$ & 67 & $6.0 \times 10^{3}$ & $1.3 \times 10^{4}$ & $0.083\left(-196^{\circ}--83^{\circ} \mathrm{C}\right)$ & $0.11\left(-83^{\circ}-21^{\circ} \mathrm{C}\right)$ \\
\hline
\end{tabular}

a Total content of TCNQ molecules by weight, $\mathrm{TCNQ}^{-}$plus $\mathrm{TCNQ}^{0}$.

Densities of $\mathrm{TCNQ}^{\circ}$ crystal and $\mathrm{BI}-n 5(\mathrm{~S})$ are estimated approximately as 1.35 and 1.20 , respectively, from the observation of their floating behavior in halogenated solvents. Consequently, the volume fraction of TCNQ molecules is slightly lower than the weight fraction.

${ }^{\mathrm{b}}$ Values in parentheses represent doping mole ratio (TCNQ ${ }^{\circ}$ TCNQ $\left.{ }^{-}\right)$.

c TCNQ salt of the ionene polymer composed of the same unit as the oligocation-TCNQ salt. These data are cited from the previous paper for comparison.

ionene polymers (IV) and LiTCNQ proceeds almost to $100 \%$, since the residual chlorine atoms are negligibly small, e.g., $0.69 \%$ and $0.80 \%$ for $\mathrm{BI}-n 5$ (S) and BI- $n 10(\mathrm{~S})$, respectively. Elemental analyses of these simple salts are consistent with the calculated values, and their visible spectra in DMF are the same with the spectrum of LiTCNQ.

\section{Conductive Properties}

The temperature dependency of the specific resistivity $\rho$ of the polymers are shown in Figures 2 and 3. The activation energies $\Delta E$ and $\rho$ of the block ionene polymers in chloride form, simple TCNQ salts and complex salts with various doping ratios are also shown in Table $I$. The activation energy was calculated by the following Arrhenius relation

$$
\rho=\rho_{0} \exp (\Delta E / k T)
$$

Specific resistivities of TCNQ salts of the block ionene polymers decrease markedly with an increase in the amount of $\mathrm{TCNQ}^{\circ}$, as in the case of TCNQ salts of ionene polymers ${ }^{5}$ reported previ- ously.

Originally, we attempted to attain desirable dielectrics by synthesizing block copolymers in which an insulating prepolymer unit is introduced into the conductive ionene-TCNQ polymer salt. If the conductive portion is completely isolated by the insulating portion in the film of block copolymers, a high specific resistivity and hence, a low dielectric loss (due to the absence of DC conduction) are to be expected in the polymers.

Contrary to this expectation, as shown in Table I, though an insulating polymer unit was introduced into the main chain, the conductivity of the TCNQ salts of the block ionenes was still high. This indicates that insulation of the conductive oligocation-TCNQ portion by polyether portion was incomplete. As shown in Table I, however, the conductivity of the TCNQ salts of the present block ionenes is lowered by introduction of the prepolymer portion when compared with that of the TCNQ salts of ionene polymers. This lowering in conductivity may be explained as a lowering 
of the TCNQ content in the block polymers. Because the content of TCNQ ${ }^{-}$in BI- $n 10(\mathrm{~S})$ is larger than that in BI- $n 5(\mathrm{~S})$, the specific resistivities of the simple and complex salts of BI- $n 10$ are smaller, although the differences are not so large, than those of BI-n5. Activation energies become smaller at low temperature and some breaks are observed in the plot of $\log \rho$ against $1 / T$. At this stage, the reason for the breaks observed in the polymers cannot be explained well, but the breaks around $-80^{\circ} \mathrm{C}$ may have some connection to the molecular motion of the polyether segment. (The glasstransition temperature of poly(oxytetramethylene) has been reported to be around $-84^{\circ} \mathrm{C}^{8}$ from dilatometric analysis.)

\section{Dielectric Properties}

The frequency dependence of a dielectric constant and a dielectric loss tangent of block ionene polymers and their simple and complex salts at various temperatures are shown in Figures 4-7. The dielectric constant increases with an increasing amount of doped $\mathrm{TCNQ}^{\circ}$. As is seen in the figures the dielectric constant increases with temperature and decreases in a high-frequency region. The values of the dielectric constants of the complex salt of BI- $n 5$, BI- $n 5(0.50)$ (doping ratio, 0.50), are from 500 to 900 and those of BI- $n 10(0.50)$ are estimated to exceed 1000 below $30 \mathrm{kHz}$ at room temperature. Such high values of dielectric constants are remarkable for organic polymers of film specimens, since the values of the dielectric constants of polymers are usually very low, ranging mostly from 2 to 10 .

The effect of the substitution of chloride anion with $\mathrm{TCNQ}^{-}$on the dielectric constant could be clearly recognized in the dielectric constant values of block ionene polymers in the chloride form $\left(\varepsilon_{\mathrm{r}} \sim\right.$ 4) and their simple TCNQ salts $\left(\varepsilon_{\mathrm{r}} \sim 6\right)$ at $-196^{\circ} \mathrm{C}$ (Figure 4). At a low temperature of $-196^{\circ} \mathrm{C}$, the electric polarization of dipoles and ionic movements is considered unlikely. Furthermore, polarization due to electronic carriers in simple salts as well seems no longer effective, since the dielectric constants of the simple salts are constant in the measuring frequency range and nearly equal to the values of the complex salts in a high frequency range and low temperature $\left(-196^{\circ} \mathrm{C}\right)$. Therefore, the difference, in regard to dielectric constants at low temperature and high frequencies, between simple salts and chloride form polymers

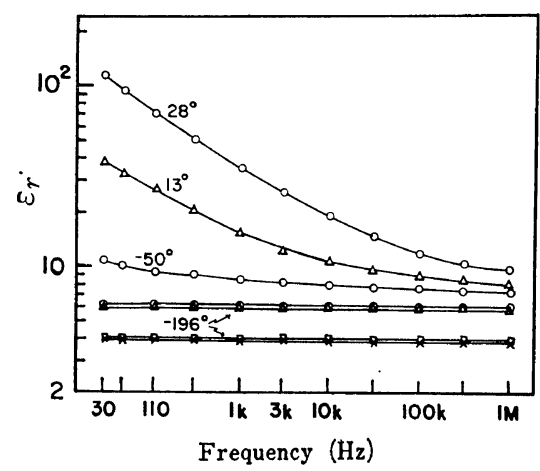

Figure 4. Plots of dielectric constant, $\varepsilon_{\mathrm{r}} v s$. frequency for $\mathrm{BI}-n 5(\times), \mathrm{BI}-n 10(\square), \mathrm{BI}-n 5(\mathrm{~S})(\triangle)$, and BI$n 10(\mathrm{~S})(\mathrm{O})$ at given temperatures.

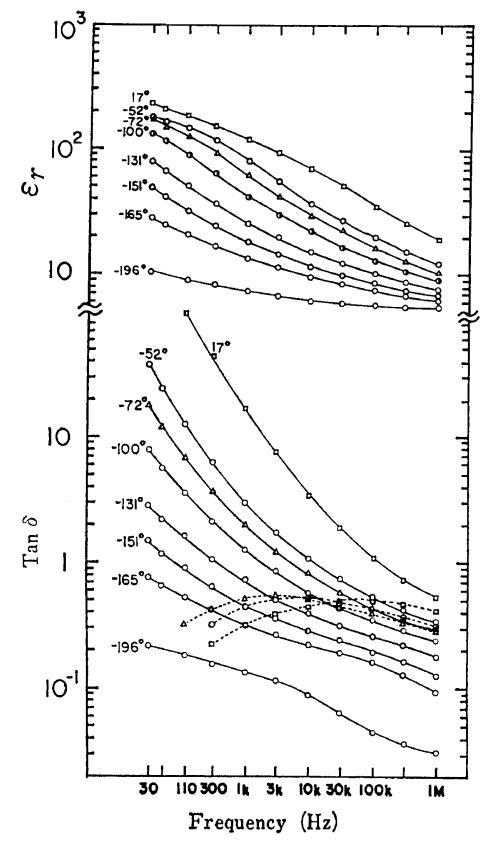

Figure 5. Plots of dielectric constant, $\varepsilon_{\mathrm{r}}$ and dielectric loss tangent, $\tan \delta v s$. frequency for $\mathrm{BI}-n 5(0.20)$ at given temperatures. The corrected $\tan \delta$ values are plotted by dotted lines.

may be due to the difference in the contribution of normal electronic and atomic polarization. ${ }^{9}$

As shown in Figures 5-7, the dispersion of dielectric constants is observed in all of the complex salts, and $\tan \delta$ maximum is observed for BI- $n 5$ $(0.50)$ and BI- $n 10(0.50)$. When the contribution of DC conductance to $\tan \delta$ is subtracted from apparent AC conductance, $\tan \delta$ maximum clearly appears for BI- $n 5(0.20)$, and shown by the dotted 


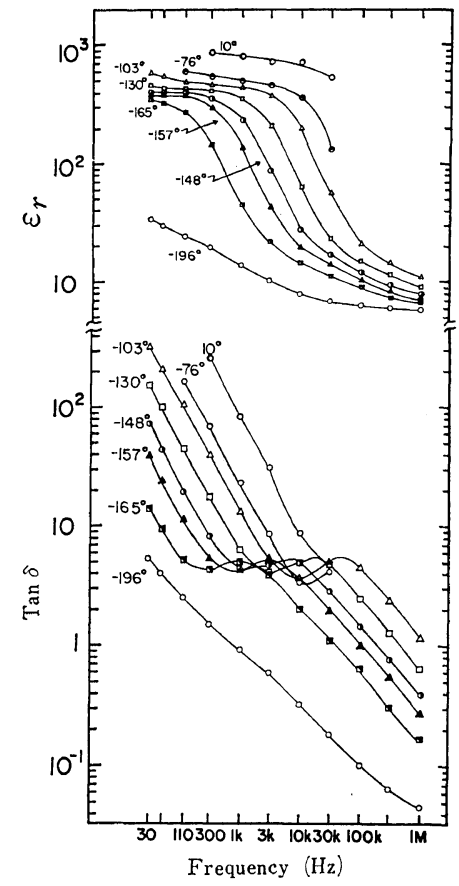

Figure 6. Plots of dielectric constant, $\varepsilon_{\mathrm{r}}$ and dielectric loss tangent, $\tan \delta v s$. frequency for $\mathrm{BI}-n 5(0.50)$ at given temperatures.

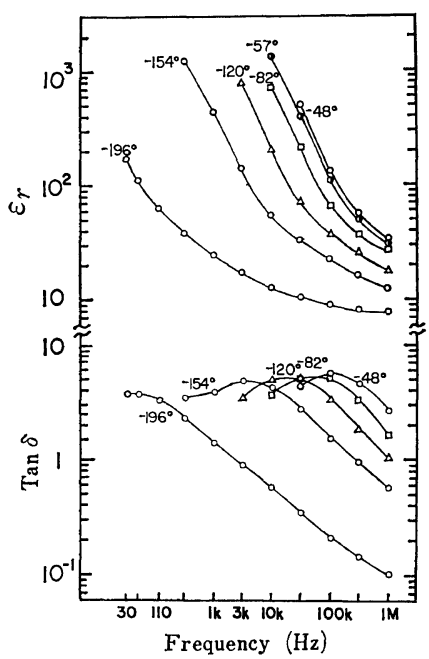

Figure 7. Plots of dielectric constant, $\varepsilon_{\mathrm{r}}$ and dielectric loss tangent, $\tan \delta$, vs. frequency for $\mathrm{BI}-n 10(0.50)$ at given temperatures.

lines in Figure 5. In the case of BI- $n 5(0.50)$ and BI- $n 10(0.50)$, relaxation frequencies shift slightly to higher frequencies when a similar correction is applied. In Figure 8, the logarithm of the re-

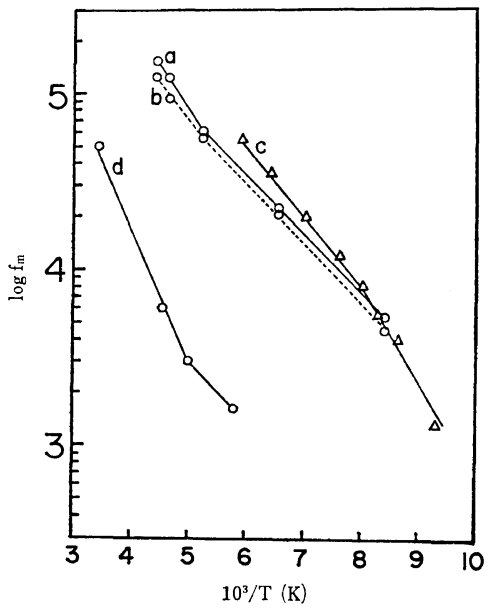

Figure 8. Plots of $\log f_{\mathrm{m}} v s \cdot 10^{3} / T$ for complex salts: (a), (b), BI- $n 10$ (0.50); (c), BI- $n 5(0.50)$; (d), BI$n 5(0.20)$. The relaxation frequencies in (a), (c), and (d) are obtained from the corrected $\tan \delta$ values, and the relaxation frequencies in (b) are obtained from $\tan \delta$ values without correction of DC conductance.

laxation frequencies $f_{\mathrm{m}}$ obtained from Figures 5-7, are plotted against the reciprocal temperature. Some breaks can be seen at the same temperature as the breaks are observed in the resistivity plots shown in Figures 2 and 3. From these curves, the activation energies $\Delta H$ associated with the polarization process could be calculated by Eyring's equation,

$$
\ln f_{\mathrm{m}}=-\frac{\Delta H}{k T}+\mathrm{const}
$$

where $k$ is Boltzmann constant. These values are shown in Table II. The activation energies for electric polarization thus obtained are in close agreement with those of the electrical conduction in the same temperature range. This suggests that both phenomena, i.e., polarization and conduction, have the same mechanistic origin: mobile-electronic-carrier generation and migration are considered to be responsible for both phenomena.

Electrical Properties in Relation to the Structural Model of Polycation-TCNQ Salts

From analogy with known crystal structures of the low-molecular cation-TCNQ salts, the structure of polycation-TCNQ salts has been, up to this point, inferred as one in which every TCNQ 
Table II. Activation energies $(\Delta H)$ of polarization process

\begin{tabular}{cll}
\hline \multicolumn{1}{c}{ Sample } & \multicolumn{1}{c}{$\Delta H, \mathrm{eV}$} \\
\hline $\mathrm{BI}-n 5(0.20)$ & $0.067\left(-100^{\circ}--72^{\circ} \mathrm{C}\right)$ & $0.15\left(-72^{\circ}-17^{\circ} \mathrm{C}\right)$ \\
$\mathrm{BI}-n 5(0.50)$ & $0.11\left(-165^{\circ}--148^{\circ} \mathrm{C}\right)$ & $0.078\left(-148^{\circ}--103^{\circ} \mathrm{C}\right)$ \\
$\mathrm{BI}-n 10(0.50)$ & $0.066\left(-154^{\circ}--82^{\circ} \mathrm{C}\right)$ & $0.10\left(-82^{\circ}-48^{\circ} \mathrm{C}\right)$ \\
$\mathrm{BI}-n 10(0.50)^{\mathrm{a}}$ & $0.067\left(-154^{\circ}--82^{\circ} \mathrm{C}\right)$ & $0.085\left(-82^{\circ}--48^{\circ} \mathrm{C}\right)$ \\
\hline
\end{tabular}

a Relaxation frequencies are obtained from $\tan \delta$ values without correction of the contribution of DC conductance.

anion radical hangs to a cationic site of a polymer chain and forms a columnar stacking of TCNQ molecules along the polymer chain with the added $\mathrm{TCNQ}^{0}$. The assumption of such a face-to-face stacking of TCNQ along the polymer chain seems to emphasize too much the regular array of TCNQ molecules in a polycation network. In the case of our TCNQ salts of block ionene polymers, the sequence of cationic sites is broken by a long unit of polyether prepolymer. Therefore, the formation of a long sequence of TCNQ along a polycation chain seems hopeless for polymers of this kind.

Considering the low regularity of cationic sites, we proposed a structural model ${ }^{5}$ of polycationTCNQ salts, in which TCNQ molecules are located randomly at electrostatically suitable positions, there being no possibility of identifying a cationic site to which some specified TCNQ belongs. The electrostatically suitable positions are those determined by the overall complicated ambient electrostatic field. Contrary to the face-to-face stacking of TCNQ along a polymer chain, every TCNQ is presumed to make "electrical contact" with several numbers of nearby TCNQ molecules, to the extent that they locate themselves in nearby positions (polyfunctionality of TCNQ molecule in electrical meaning). Thus, such an electrical contact through nearby TCNQ molecules would form a conductive sequence of TCNQ (conduction path), differing considerably from the hitherto considered TCNQ columns involving the face-toface stacking of TCNQ.

The presence of three types of conduction paths has been inferred in the model: (1) a continuous path; the path is continuous from the electrode of one side to that of the other side, which contributes to DC conductivity, (2) a branched path; one end of this path starts from the electrode of one side, but ends at some place in-between the electrodes, and (3) an isolated path; both ends of this path exist in the bulk. Both branched and isolated paths can contribute to electric polarization but not to DC conductivity. The fraction of three paths is naturally an important factor, but the nature of the conduction path is also another important factor which determines the electrical properties of the polymers. The nature of the conduction path determines the degree of ease of mobile-carrier generation and migration in the conduction path; this nature is considered to depend mainly on the species of the interacting pair of the TCNQ, i.e., $\mathrm{TCNQ}^{-}$... TCNQ $\mathrm{TC}^{-}$or $\mathrm{TCNQ}^{-}$ $\cdots \mathrm{TCNQ}^{0}$. The mobile-carrier generation and migration must be easier in complex salts than in simple salts in which the Coulomb repulsive forces have to be overcome to form the dianion state transiently. ${ }^{10}$ Actually, this difference is clearly observed in the electrical properties of the polymers in the simple salt form and complex salt form.

As described in a previous paper, ${ }^{5}$ the estimation of the fraction of various paths in the material is possible by applying the mathematical method developed by P. J. Flory ${ }^{11}$ explaining the polymerization reaction of polyfunctional monomers. In order to apply this to our case, the polyfunctionality of the monomers must be replaced with the "electrical" functionality of a TCNQ molecule. This electrical functionality of TCNQ molecule is defined as the maximum number of the sites, which exist in nearby positions of the TCNQ molecule such that, if these sites are each occupied by a TCNQ entity, the TCNQ molecules can make electrical contact with the central TCNQ in question. The probability of forming "electrical contact" between nearby TCNQ is considered a probability of occupation of nearby sites of a given TCNQ, and this probability must be replaced in our case by the extent of reaction $\alpha$ in Flory's theory. Consequently, the number of nearby TCNQ in contact with a TCNQ is smaller than the 
number of the functionality of a TCNQ. In this case, we may assume this probability of forming electrical contact to be equal to the volume fraction of TCNQ. As discussed in a previous paper in detail, when the volume fraction of TCNQ $\alpha$ is smaller than some critical value, the conduction path formed by a sequence of electrically contacted TCNQ molecules is an exclusively isolated one, while, when $\alpha$ exceeds this critical value, continuous conduction paths begin to form. Therefore, both isolated and continuous paths should coexist in the material where $\alpha$ exceeds the critical value. The fraction ratio of continuous path against the isolated path increases with an increase in $\alpha$. Assuming the functionality of TCNQ to be four, the fraction of various paths with the volume fraction of TCNQ $\alpha$ can be calculated using Flory's equation. The results are shown in Figure 9. Considering the rather high content of TCNQ, (Table I), both the continuous and isolated paths are expected to coexist in the polymers. This result is consistent with the high conductivity and high dielectric constant of the present polymers.

Electrically, such a system can be illustrated by an equivalent network shown in Figure 10. In Figure 10A, each conduction path is represented simply by a resistor. As to the extent that the conduction path consists of organic materials, it should have some capacitance determined by the geometrical factor of the path and the intrinsic dielectric constant of the path. This intrinsic dielectric constant is the one determined mainly by normal electronic polarization and atomic polarization, and also, any orientation polarization, occurring in the path. The contribution of

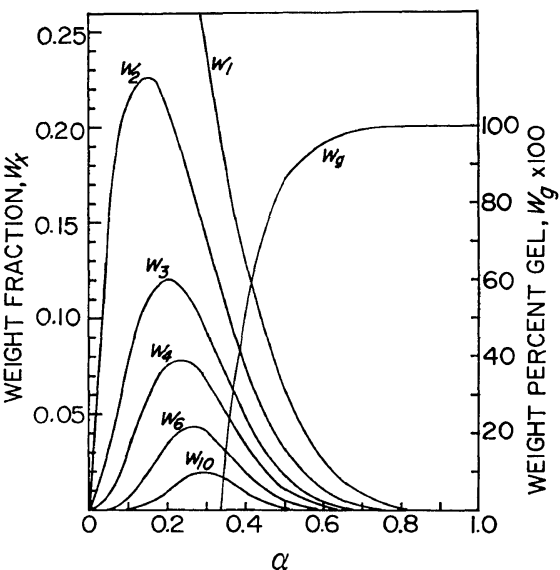

Figure 9. Weight fractions of various paths as a function of volume fraction of TCNQ molecules, $\alpha$ (functionality of TCNQ molecules, $f=4$ ).

this capacitance of each conduction path on the total film capacitance observed is considered very small when compared with that from interfacial polarization induced by the migration of mobile electronic carriers. Therefore, in order to clearly illustrate the contribution of electronic carriers on the interfacial polarization, the capacitance of the path is ignored in Figure 10A. For clarity in this figure, the continuous path represented by (a) contributes only DC conduction, and all of the branched path (b) and isolated paths (c), (d) contribute to the electric polarization. The electrical properties of the polymers will depend on the fraction of these various paths and nature of the conduction paths (i.e., the resistivity of the resistor). The electric polarization induced by (b), (c), and (d) may be understood as the electrode

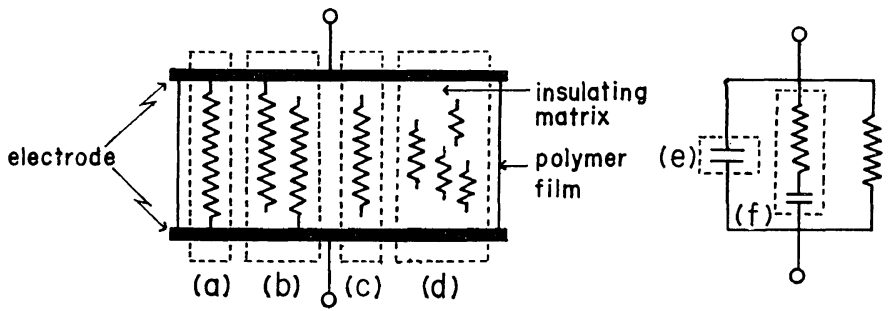

Figure 10. Schematic representation of equivalent network for polycation-TCNQ salt. (A) Representation of various conduction paths by resistors. The capacitance of the paths is ignored in the figure: (a), continuous path; (b), branched path; (c) and (d), isolated path.

(B) Equivalent network system explaining the frequency dependence of the dielectric properties of the material: (e), the capacitance due to the insulating matrix; (f), the capacitance due to the polarization induced by (b), (c), and (d), including the gap capacitance. 
and interfacial polarization, respectively. It must be noted that some capacitance, which may be called gap capacitance, will also exist between paths shown typically by (b). Considering the existence of the vast number of the paths in the material, the contribution of the gap capacitance could not be ignored. The frequency dependence of the polymers may be clearly demonstrated by rewriting (A) as (B). As mentioned above, since the capacitance of the conduction path is ignored, a capacitor (e) in Figure 10B represents the capacitance due to the insulating matrix. The element represented by (f) in Figure 10B represents the polarization induced by (b), (c), and (d), including the gap capacitance.

The material represented in Figure 10A may be considered as the dielectric with microscopic heterogeneity with conducting particles dispersed in an insulating matrix. The dielectric properties of such a material could be analized by the theory developed by Maxwell, Wagner and Sillars. The relaxation frequency, $f_{\mathrm{m}}$ of Maxwell-Wagner type polarization is given ${ }^{12}$ as follows:

$$
f_{\mathrm{m}}=\frac{2 \sigma_{2}}{(n-1) \varepsilon_{1}+\varepsilon_{2}}
$$

where $\varepsilon_{1}$ and $\varepsilon_{2}$ are dielectric constant of insulating matrix and conducting particles, respectively, and $\sigma_{2}$, conductivity of the particles, and $n$, a shape factor of the conducting particles. The dielectric constant $\varepsilon_{2}$ in the case of the conducting particles corresponds with the intrinsic dielectric constant in the case of the conduction path mentioned above. Since the change of $\varepsilon_{1}$ and $\varepsilon_{2}$ with temperature is considered negligible when compared with the change of $\sigma_{2}$, the change of $f_{\mathrm{m}}$ with temperature depends mainly on the change of $\sigma_{2}$. If such a microscale Maxwell-Wagner type polarization is effective in our polymers, linear relation should be expected in the plot of $\log f_{\mathrm{m}} v s . \log \sigma_{2}$. As the observed conductivity $\sigma$ of the material may be considered to be proportional to the conductivity of the conducting particles, namely $\sigma_{2}, \log \sigma$, instead of $\log \sigma_{2}$, is plotted against $\log f_{\mathrm{m}}$ in Figure 11, at different temperatures for the complex salts. The linear relation is actually obtained in all plots, and the slopes of the respective lines are approximately $45^{\circ}$. Thus, the nature of the electric polarization of the TCNQ salts of block ionene polymers is explainable qualitatively by the microscale

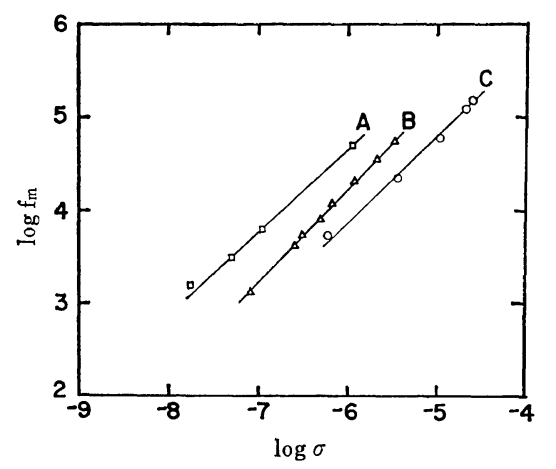

Figure 11. Plots of $\log$ relaxation frequency, $f_{\mathrm{m}} v s$. $\log$ DC conductivity, $\sigma$ for the complex salts. The relaxation frequencies are obtained by the frequency dependence of corrected $\tan \delta$ : (A), BI- $n 10(0.50)$; (B), BI-n5 (0.50); (C), BI-n5 (0.20).

Maxwell-Wagner type polarization mechanism.

In this study, several new TCNQ salts of block ionene polymers were synthesized, and their conductive and dielectric properties were measured. In these polymers, long columnar stacking of TCNQ molecules were impossible, since polycation sequences are broken by the long unit of polyether prepolymer. In picturing and explaining the structure and electrical properties of the TCNQ salts of such block ionene polymers, the structural model of the polycation-TCNQ salts proposed in a previous paper ${ }^{5}$ is applicable. With this model, the anomalous high dielectric constant of the polymers were explained qualitatively by a microscale Maxwell-Wagner type polarization mechanism.

Acknowledgement. One of the authors (S. I.) is grateful to the Managing Director of the Matsushita Electric Works Co. Ltd., K. Kobayashi and the Manager of Chemical Products Section, T. Murakami for giving him the opportunity of carrying out this work at Osaka University.

\section{REFERENCES}

1. J. E. Katon, "Organic Semi-conducting Polymers," Dekker, New York, N. Y., 1968.

2. Ya.M. Paushkin, T.P. Yishnyakova, A.F. Lunin, and S. A. Nizova, "Organic Polymeric Semiconductors," John Wiley and Sons, New York, N.Y., 1974.

3. J. H. Lupinski, K. D. Kopple, and L. J. Hertz, J. Polym. Sci., Part C, 16, 1561 (1967). 
4. K. Mizoguchi, T. Suzuki, E. Tsuchida, and I. Shinohara, Nippon Kagaku Kaishi (J. Chem. Soc. Jpn. Chem. Ind. Chem.), 1973, 1760.

5. S. Ikeno, K. Matsumoto, M. Yokoyama, and H. Mikawa, Polym. J., 9, 261 (1977).

6. S. Somoano, S.P.S. Yen, and A. Rembaum, $J$. Polym. Sci., Polym. Lett. Ed., 8, 467 (1970).

7. K. Akagi, S. Oae, and M. Murakami, J. Am.
Chem. Soc., 79, 3118 (1957).

8. R. E. Wetton and G. Williams, Trans. Faraday Soc., 61, 2132 (1965).

9. This argument was suggested by the referee.

10. O. Leblanc, J. Chem. Phys., 42, 4307 (1965).

11. P. J. Flory, "Principles of Polymer Chemistry," Conell Univ. Press, Ithaca, N.Y., 1953, Chap. 9.

12. R. W. Sillars, J.I.E.E., 80, 378 (1937). 